\title{
Quantifying entanglement with scattering experiments
}

\author{
O. Marty,,${ }^{1,2}$ M. Epping, ${ }^{3}$ H. Kampermann, ${ }^{3}$ D. Bruß, ${ }^{3}$, M.B. Plenio,,${ }^{1,2}$ and M. Cramer ${ }^{1,2}$ \\ ${ }^{1}$ Institut für Theoretische Physik, Albert-Einstein Allee 11, Universität Ulm, D-89069 Ulm, Germany \\ ${ }^{2}$ Institute for Integrated Quantum Science and Technology, \\ Albert-Einstein Allee 11, Universität Ulm, D-89069 Ulm, Germany \\ ${ }^{3}$ Institute for Theoretical Physics III, Heinrich-Heine-Universität Düsseldorf, Universitätsstr. 1, 40225 Düsseldorf, Germany
}

\begin{abstract}
We show how the entanglement contained in states of spins arranged on a lattice may be quantified with observables arising in scattering experiments. We focus on the partial differential cross-section obtained in neutron scattering from magnetic materials but our results are sufficiently general such that they may also be applied to, e.g., optical Bragg scattering from ultracold atoms in optical lattices or from ion chains. We discuss resonating valence bond states and ground and thermal states of experimentally relevant models - such as Heisenberg, Majumdar-Ghosh, and XY models - in different geometries and with different spin numbers. As a by-product, we find that for the one-dimensional XY model in a transverse field such measurements reveal factorization and the quantum phase transition at zero temperature.
\end{abstract}

\section{INTRODUCTION}

Entanglement is a key resource for performing quantum information tasks [1,2]. At low temperatures, it occurs naturally in quantum many-body systems and its amount (more concretely, its scaling with the size of system partitions) relates to the complexity of descriptions of such systems [3-7]. It also serves to characterize exotic states of matter, a prominent example being topological spin liquids, see, e.g., the recent Refs. [8, 9]. While the task of merely verifying that entanglement is present $[10,11]$ is quite established and has been demonstrated in a number of experiments [12-22], quantifying its amount rigorously and without any assumptions is a delicate task and has only very recently been experimentally achieved for a large many-body system of bosons in optical lattices in Ref. [23] (see also, e.g., Ref. [24] where the entanglement of a small photonic system was quantified using few measurements). Generally speaking, the difficulty increases with the number of particles carrying the quantum information, i.e., it is especially delicate for large systems for which the available measurements are usually very limited and very far from being informationally complete (in which case full state tomography $[15,25,26]$ would be possible). Here, we are interested in such large systems, namely a large number of spins arranged on a lattice. In order to quantify the amount of entanglement that is shared between the spins, we rely only on global measurements typically obtained in scattering experiments. We achieve this by generalizing results of the recent Refs. [27, 28] to arbitrary spin and to more general observables. In the case of neutron scattering from magnetic materials, this enables us to quantify entanglement for arbitrary lattice geometries relying solely on the Fourier transform of the scattering cross-section (or, alternatively, measurements that do not resolve the energy of the scattered neutrons). Our strategy adopts a principle from quantum information theory that is simple yet powerful [29-33]: Given certain observables and their experimentally obtained expectation values, we ask what is the minimal amount of entanglement that is consistent with the obtained outcomes, i.e., given the expectation values of the observables, we minimize over all density matrices that are consistent with them. In this way, we arrive at the least amount of entanglement that is consistent with the measurement outcomes and thus we put a lower bound on the entanglement contained in the sample on which the measurements were performed. By the very nature of this principle, we need not make any assumptions on the system (such as, e.g., the temperature, details of external potentials, the Hamiltonian governing the system, or even the system being in equilibrium).

We consider observables that arise in scattering experiments from $N$ spins arranged on a lattice. Examples include optical Bragg scattering from ultracold atoms in optical lattices [34] or from ion chains [35] and neutron scattering from magnetic materials [36]. These observables may be written as

$$
\hat{S}(\boldsymbol{q})=\sum_{\alpha, \beta} M_{\alpha, \beta}(\boldsymbol{q}) \hat{S}_{\alpha, \beta}(\boldsymbol{q}),
$$

where, usually, $\boldsymbol{q}=\boldsymbol{k}_{f}-\boldsymbol{k}_{i}$ is the scattering vector, i.e. the difference between the final and the initial wave vector. Here,

$$
\hat{S}_{\alpha, \beta}(\boldsymbol{q})=\sum_{i, j=1}^{N} f_{i, \alpha}^{*}(\boldsymbol{q}) f_{j, \beta}(\boldsymbol{q}) \mathrm{e}^{\mathrm{i} \boldsymbol{q}\left(\boldsymbol{r}_{i}-\boldsymbol{r}_{j}\right)} \hat{S}_{i}^{\alpha} \hat{S}_{j}^{\beta},
$$

where $\boldsymbol{r}_{i}$ is the position of the $i$ 'th spin with corresponding spin operators $\hat{S}_{i}^{\alpha}, \alpha=x, y, z$, and spin quantum number $s$, and the coefficients $M_{\alpha, \beta}(\boldsymbol{q})$ and $f_{i, \alpha}(\boldsymbol{q})$ depend on the system under consideration. While keeping our results as general as possible, we will focus on neutron scattering experiments, in which such observables arise as follows.

The neutrons interact magnetically with the atoms of the target sample, whose magnetic moments mostly originate from the orbital motion and spins of unpaired electrons. In many cases an effective spin value can be assigned to either the magnetic atoms or to the entire unit-cell [36]. With the formalism introduced by Van Hove in Ref. [37], the partial differential cross-section can be expressed in terms of timedependent correlation functions. Accordingly, for unpolarized neutrons, the magnetic cross-section is proportional to [36]

$$
\begin{aligned}
\frac{k_{f}}{k_{i}} \sum_{\alpha, \beta}\left(\delta_{\alpha, \beta}-\bar{q}_{\alpha} \bar{q}_{\beta}\right) \sum_{i, j} f_{i, \alpha}(\boldsymbol{q})^{*} f_{j, \beta}(\boldsymbol{q}) \mathrm{e}^{\mathrm{i} \boldsymbol{q}\left(\boldsymbol{r}_{i}-\boldsymbol{r}_{j}\right)} \\
\times \int \mathrm{d} t \mathrm{e}^{-\mathrm{i} \omega t}\left\langle\hat{S}_{i}^{\alpha} \hat{S}_{j}^{\beta}(t)\right\rangle,
\end{aligned}
$$


where $\omega$ is the energy transferred to the sample. Furthermore, $f_{i, \alpha}(\boldsymbol{q})=F_{i}(\boldsymbol{q}) g_{i, \alpha}$, where $F_{i}(\boldsymbol{q})$ and $g_{i, \alpha}$ denote the magnetic form factor and the Landé factor of the $i$ 'th site, respectively, and $\overline{\boldsymbol{q}}=\boldsymbol{q} /|\boldsymbol{q}|$. In general, we allow the g-factor to be anisotropic and $f_{i, \alpha}(\boldsymbol{q})$ to be site-dependent, where $i$ labels the lattice sites with corresponding effective values of $f_{i, \alpha}$ and $\hat{S}_{i}^{\alpha}$ (corresponding to an effective spin quantum number $s$ ). The magnetic form factor $F_{i}(\boldsymbol{q})$ stems from the finite extent of the electron orbitals seen by the neutron with wavelength of the order of interatomic distances. To determine it, a detailed knowledge about the electronic wave functions of the magnetic atoms in the scatterer is required, and its values may be found in the literature. As $k_{i, f}$ are known, one may multiply (3) by $k_{i} / k_{f}$ and take the Fourier transform to obtain the instantaneous scattering function $S(\boldsymbol{q})=\langle\hat{S}(\boldsymbol{q})\rangle$, where $\hat{S}(\boldsymbol{q})$ is as in Eq. (1) with $M_{\alpha, \beta}(\boldsymbol{q})=\delta_{\alpha, \beta}-\bar{q}_{\alpha} \bar{q}_{\beta}$. Alternatively, $S(\boldsymbol{q})$ may be obtained if the requirements of the static approximation are fulfilled [38] and the final energy is not resolved. For quasi-one- or two-dimensional systems one may also consider a special scattering geometry [39-41] to obtain $S(\boldsymbol{q})$.

In Section II, we show how a lower bound to the entanglement shared among $N$ spins-as quantified in terms of the best separable approximation [42] or the (generalized) robustness of entanglement $[43,44]$ - may be obtained from the expectation value of $\hat{S}(\boldsymbol{q})$ in Eq. (1). In this way, we quantify entanglement of a collection of $N$ spins without any assumption on the system. In Sections III and IV we show that our method allows to quantify the entanglement of ground and thermal states corresponding to several model Hamiltonians. We conclude with a summary and outlook in Section V.

\section{MAIN RESULTS}

In this section we will show how observables as in Eq. (1) may serve as lower bounds to the entanglement. We will consider several entanglement monotones and a particular simple form will be derived for the best separable approximation $\mathcal{E}_{B S A}[\hat{\varrho}]$ in the neutron scattering setting: For any scattering vector $\boldsymbol{q}$, we find (see below and Appendix A for details)

$$
\mathcal{E}_{B S A}[\hat{\varrho}] \geq 1-\frac{1}{c_{\min }} \sum_{\alpha, \beta}\left(\delta_{\alpha, \beta}-\bar{q}_{\alpha} \bar{q}_{\beta}\right)\left\langle\hat{S}_{\alpha, \beta}(\boldsymbol{q})\right\rangle,
$$

where $c_{\min }$ is a constant that depends on the spin quantum number $s$ and the magnetic form factors $F_{i}(\boldsymbol{q})$ and Landé factors $g_{i, \alpha}$. Hence, a measurement of the Fourier transform of the magnetic scattering cross-section at a single scattering vector directly provides a lower bound to the entanglement contained in the sample. A numerical analysis of the above bound may be found in Section III (see Figs. 1-3) for different physical models that describe, among others, the magnetic compounds summarized in Table 1.

In the remainder of this section, we detail the derivation of the above bound and the bounds on robustness of entanglement measures. We start with a detailed description of the scattering observables under consideration.

\section{A. The observables under consideration}

We will see below that for many systems, a measurement of $\langle\hat{S}(\boldsymbol{q})\rangle$ at a single scattering vector $\boldsymbol{q}$ suffices to put meaningful tight lower bounds on the entanglement quantified via the best separable approximation (BSA). For the robustness measures, however, we have found that measurements at a single scattering vector $\boldsymbol{q}$ do not suffice to obtain non-trivial bounds for large systems (see also Ref. [27]). To this end, we incorporate knowledge of $\langle\hat{S}(\boldsymbol{q})\rangle$ at several $\boldsymbol{q}$ by slightly generalizing the observables in the introduction to observables of the form

$$
\hat{S}=\sum_{\boldsymbol{q} \in Q} \hat{S}(\boldsymbol{q})
$$

As we will see, this summation over measurements obtained at several scattering vectors will result in positive entanglement bounds even in the thermodynamic limit. Here, $Q \subset \mathbb{R}^{3}$ is some collection of scattering vectors and $\hat{S}(\boldsymbol{q})$ is defined as in Eq. (1), where we make the following assumptions on the coefficients $M_{\alpha, \beta}(\boldsymbol{q}) \in \mathbb{C}$ and $f_{i, \alpha}(\boldsymbol{q}) \in \mathbb{C}$ : We assume that the $3 \times 3$ matrix $M(\boldsymbol{q})$ with entries $M_{\alpha, \beta}(\boldsymbol{q})$ is Hermitian, i.e., $M_{\alpha, \beta}(\boldsymbol{q})=M_{\beta, \alpha}^{*}(\boldsymbol{q})$, and positive semi-definite. We further assume that for each $i=1, \ldots, N$ the $3 \times 3$ matrix $M^{(i)}$ with entries

$$
M_{\alpha, \beta}^{(i)}=\sum_{\boldsymbol{q} \in Q} f_{i, \alpha}^{*}(\boldsymbol{q}) f_{i, \beta}(\boldsymbol{q}) M_{\alpha, \beta}(\boldsymbol{q})
$$

is real and symmetric, i.e., $M_{\alpha, \beta}^{(i)}=M_{\beta, \alpha}^{(i)} \in \mathbb{R}$. All these assumptions are fulfilled, e.g., in the neutron scattering setting, for which we have $M(\boldsymbol{q})=\mathbb{1}-\overline{\boldsymbol{q}} \overline{\boldsymbol{q}}^{t}$ (see Eq. (3)) and $f_{i, \alpha}(\boldsymbol{q})=F_{i}(\boldsymbol{q}) g_{i, \alpha}$ with $g_{i, \alpha} \in \mathbb{R}$.

\section{B. Lower bounds to the entanglement}

In what follows, we consider multipartite entanglement in the following sense. Every state $\hat{\varrho}$ that is not fully separable, i.e., of the form

$$
\sum_{n} p_{n} \bigotimes_{i=1}^{N} \hat{\varrho}_{i}^{(n)} \in \mathcal{S},
$$

with $p_{n}>0$ and $\sum_{n} p_{n}=1$, will be called entangled. Here, we denoted the set of separable states by $\mathcal{S}$. The degree of entanglement is then quantified using entanglement monotones $[1,2]$, that is, functionals $\mathcal{E}[\hat{\varrho}]$ that do not increase under local operations and classical communication. The monotones under consideration are part of a larger family of monotones that may be expressed as [30]

$$
\mathcal{E}_{\mathcal{C}}[\hat{\varrho}]=-\min _{\hat{W} \in \mathcal{W} \cap \mathcal{C}} \operatorname{tr}[\hat{W} \hat{\varrho}]
$$

with the convention that $\mathcal{E}_{\mathcal{C}}[\hat{\varrho}]=0$ if the minimization results in a positive number. Here, $\mathcal{W}$ is the set of entanglement witnesses (Hermitian operators with non-negative expectation 
value for every separable state, i.e., $\langle\hat{W}\rangle_{\text {sep. }} \geq 0$, see Ref. [11] for a review) and the set $\mathcal{C}$ depends on the chosen entanglement measure: If

$$
\mathcal{C}=\{\hat{W} \in \mathcal{W} \mid \mathbb{1}+\hat{W} \geq 0\}
$$

then $\mathcal{E}_{\mathcal{C}}[\hat{\varrho}]=\mathcal{E}_{B S A}[\hat{\varrho}]$ quantifies entanglement in terms of the best separable approximation [42], which, in essence, answers the question of how much of a separable state is contained in the state $\hat{\varrho}$. For

$$
\mathcal{C}=\{\hat{W} \in \mathcal{W} \mid \operatorname{tr}[\hat{W} \hat{\sigma}] \leq 1 \forall \hat{\sigma} \in \mathcal{S}\}
$$

we have $\mathcal{E}_{\mathcal{C}}[\hat{\varrho}]=\mathcal{E}_{R}[\hat{\varrho}]$, quantifying entanglement in terms of the robustness of entanglement. Finally, if

$$
\mathcal{C}=\{\hat{W} \in \mathcal{W} \mid \mathbb{1}-\hat{W} \geq 0\}
$$

then $\mathcal{E}_{\mathcal{C}}[\hat{\varrho}]=\mathcal{E}_{G R}[\hat{\varrho}]$ is the generalized robustness of entanglement. These robustness measures $[43,44]$ quantify the minimal amount of noise (in the form of general state in the case of the generalized robustness and in the form of a separable state in the case of the robustness) that must be mixed in to make $\hat{\varrho}$ separable.

Instead of minimizing over all the entanglement witnesses $\hat{W} \in \mathcal{W} \cap \mathcal{C}$, we construct a single member of the set $\mathcal{W} \cap \mathcal{C}$ of the form

$$
\hat{W}_{\hat{S}, \mathcal{C}}=a_{\mathcal{C}} \hat{S}+b_{\mathcal{C}} \mathbb{1}
$$

with appropriate real coefficients $a_{\mathcal{C}}$ and $b_{\mathcal{C}}$ (which will depend on the set of scattering vectors $Q$ and the matrices $M(\boldsymbol{q})$ ) and $\hat{S}$ as in the previous section. By inspection of Eq. (8), we see that any $\hat{W} \in \mathcal{W} \cap \mathcal{C}$ gives a lower bound to the entanglement monotone and thus for any state $\hat{\varrho}$, one has

$$
\mathcal{E}_{\mathcal{C}}[\hat{\varrho}] \geq-a_{\mathcal{C}}\langle\hat{S}\rangle-b_{\mathcal{C}},
$$

which depends only on the expectation value $\langle\hat{S}\rangle=\operatorname{tr}[\hat{S} \hat{\varrho}]$. The coefficients are found in the following way. As the matrices $M(\boldsymbol{q})$ are assumed to be positive semidefinite, it is straightforward to show that $\hat{S}$ is also positive semidefinite, see Appendix A. Furthermore, one may derive bounds on the minimal and maximal achievable expectation value in fully separable states

$$
c_{\min } \leq\langle\hat{S}\rangle_{\text {sep. }} \leq c_{\max } .
$$

Together with positive semidefiniteness of $\hat{S}$, such bounds allow us to arrive at witnesses that are of the form as in Eq. (12) and members of the set $\mathcal{W} \cap \mathcal{C}$. One readily verifies that the coefficients

$$
\begin{aligned}
a_{B S A} & =\frac{1}{c_{\min }}, \quad b_{B S A}=-1, \\
a_{R} & =-\frac{1}{c_{\max }-c_{\min }}, \quad b_{R}=-c_{\max } a_{R}, \\
a_{G R} & =-\frac{1}{c_{\max }}, \quad b_{G R}=1,
\end{aligned}
$$

fulfil the necessary requirements as defined in Eqs. (9-11). It remains to make the bounds $c_{\min }$ and $c_{\max }$ explicit.

\section{Lower bound to the best separable approximation}

For each $i=1, \ldots, N$, denote the eigenvalues of the $3 \times 3$ matrix $M^{(i)}$ in Eq. (6) by $m_{\alpha}^{(i)}$. For product states, so for each summand in Eq. (7), the expectation value $\left\langle\hat{S}_{i}^{\alpha} \hat{S}_{j}^{\beta}\right\rangle$ can be written as the product $\left\langle\hat{S}_{i}^{\alpha}\right\rangle\left\langle\hat{S}_{j}^{\beta}\right\rangle$ for lattice sites $i \neq j$. The resulting expression can then be bounded with the help of the eigenvalues of the coefficient matrices $M^{(i)}$ in the following way (see Appendix A for details).

$$
c_{\min }=\sum_{i=1}^{N} \min _{|\psi\rangle} \sum_{\alpha} m_{\alpha}^{(i)}\left(\left\langle\psi\left|\hat{S}_{\alpha}^{2}\right| \psi\right\rangle-\left\langle\psi\left|\hat{S}_{\alpha}\right| \psi\right\rangle^{2}\right),
$$

where $\hat{S}_{\alpha}, \alpha=x, y, z$, are the spin operators for a single spin. For each $i$, the minimization over pure states $|\psi\rangle \in \mathbb{C}^{2 s+1}$ may be solved numerically. For some special cases, it may be given explicitly: E.g., for $f_{i, \alpha}(\boldsymbol{q})=f(\boldsymbol{q})$ and $M_{\alpha, \beta}(\boldsymbol{q})=$ $\delta_{\alpha, \beta} /|f(\boldsymbol{q})|^{2}$ (similar observables were considered in [28]), one finds

$$
c_{\min }=N|Q| s,
$$

where we recall that $s$ is the spin quantum number corresponding to the $\hat{S}_{i}^{\alpha}$ and $|Q|$ denotes the number of scattering vectors in the set $Q$. If $f_{i, \alpha}(\boldsymbol{q})=f(\boldsymbol{q})$, if $M(\boldsymbol{q})=\left(\mathbb{1}-\overline{\boldsymbol{q}} \overline{\boldsymbol{q}}^{t}\right) /|f(\boldsymbol{q})|^{2}$ as in the neutron scattering setting, and if $Q$ contains only one scattering vector, we have

$$
c_{\text {min }}=N C_{s},
$$

where [45]

$$
C_{s}= \begin{cases}\frac{1}{4} & \text { for } s=\frac{1}{2}, \\ \frac{7}{16} & \text { for } s=1,\end{cases}
$$

and further values are listed in Ref. [46]. The latter yields the following bound on the best separable approximation. For each $\boldsymbol{q} \in \mathbb{R}^{3}$, inserting Eq. (18) into Eqs. (13) and (15) leads to

$$
\begin{aligned}
\mathcal{E}_{B S A}[\hat{\varrho}] & \geq 1-\sum_{\alpha, \beta} \frac{\delta_{\alpha, \beta}-\bar{q}_{\alpha} \bar{q}_{\beta}}{N C_{s}} \sum_{i, j} \mathrm{e}^{\mathrm{i} \boldsymbol{q}\left(\boldsymbol{r}_{i}-\boldsymbol{r}_{j}\right)}\left\langle\hat{S}_{i}^{\alpha} \hat{S}_{j}^{\beta}\right\rangle \\
& =: E_{B S A}[\hat{\varrho}](\boldsymbol{q}) .
\end{aligned}
$$

Note that this is a general bound for any state. Whenever the expectation value $E_{B S A}[\varrho](\boldsymbol{q})$ is accessible, it provides a lower bound to the entanglement contained in $\hat{\varrho}$ - no matter what the underlying Hamiltonian of the system or the temperature might be, no matter whether the system is in equilibrium or not. If, depending on the experimental situation, $E_{B S A}[\hat{\varrho}](\boldsymbol{q})$ is not accessible, i.e. the special form of $M(\boldsymbol{q})$ and $f_{i, \alpha}(\boldsymbol{q})$ is not given, one has to use the observable given in Eqs. (1) and (2) and the general bound in Eq. (16) needs to be applied. Note that, for any state, $\mathcal{E}_{B S A}[\hat{\varrho}] \leq 1$, i.e., whenever we find $E_{B S A}[\hat{\varrho}](\boldsymbol{q})=1$, the bound is in fact equal to the exact entanglement. In Section III, we present $E_{B S A}[\hat{\varrho}](\boldsymbol{q})$ for several numerically simulated states, see Figs. 1-3, and in Section IV, we discuss some examples for which $E_{B S A}[\hat{\varrho}](\boldsymbol{q})$ may be obtained analytically. 

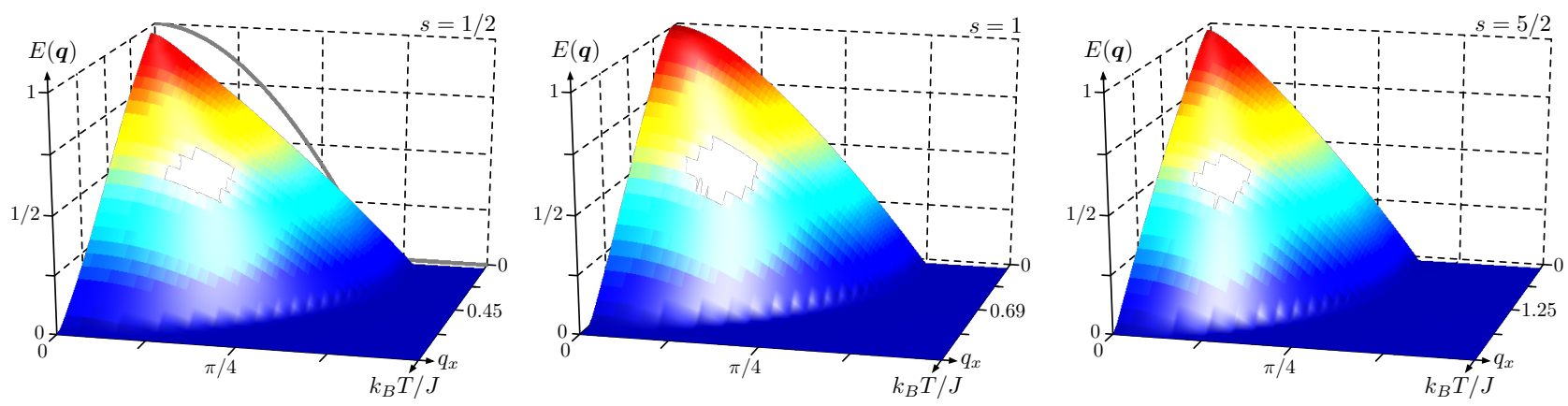

FIG. 1. Lower bound $E_{B S A}[\hat{\varrho}](\boldsymbol{q})=E_{B S A}[\hat{\varrho}]\left(q_{x}\right)$ (Eq. (20)) to the entanglement $\mathcal{E}_{B S A}[\hat{\varrho}]$ vs. temperature for thermal states of the quasione-dimensional Heisenberg model Eqs. $(26,27)$ for $s=1 / 2,1,5 / 2$ (left to right) and $L=900$ [68]. The gray solid line in the left-most plot depicts the entanglement bound for a ground state of the Majumdar-Gosh model in the limit $L \rightarrow \infty$. Note that for all shown models the bound $\mathcal{E}_{B S A}[\hat{\varrho}] \leq 1$, which holds for any state $\hat{\varrho}$, is attained at $T=0, q_{x}=0$.

\section{Lower bound to robustness measures}

The derivation of the general bound may be found in Appendix A, for clarity, we only state it here for the following special case. We let $f_{i, \alpha}(\boldsymbol{q})=f(\boldsymbol{q})$ and $M_{\alpha, \beta}(\boldsymbol{q})=$ $\delta_{\alpha, \beta} /|f(\boldsymbol{q})|^{2}$ such that our observable reads

$$
\hat{S}=\sum_{\boldsymbol{q} \in Q} \sum_{i, j=1}^{N} \mathrm{e}^{\mathrm{i} \boldsymbol{q}\left(\boldsymbol{r}_{i}-\boldsymbol{r}_{j}\right)} \sum_{\alpha} \hat{S}_{i}^{\alpha} \hat{S}_{j}^{\alpha}
$$

We further assume that the $N=N_{1} N_{2} N_{3}$ spins are arranged on a finite three-dimensional Bravais lattice with primitive vectors $\boldsymbol{a}_{d}, d=1,2,3$, such that $\boldsymbol{r}_{i}=\sum_{d=1}^{3} i_{d} \boldsymbol{a}_{d}$ with $i_{d} \in\left\{1, \ldots, N_{d}\right\}$. Further we assume that

$$
Q \subset\left\{\sum_{d=1}^{3} q_{d} \boldsymbol{b}_{d} \mid q_{d} \in \frac{1}{N_{d}}\left\{0, \ldots, N_{d}-1\right\}\right\}=: \mathcal{Q}
$$

where the $\boldsymbol{b}_{d}$ are the reciprocal primitive vectors. The upper bound is derived in appendix $\mathrm{A}$ and reads

$$
c_{\max }=N|Q| s+N^{2} s^{2}
$$

see Eq. (A17). Hence, whenever the expectation of the observable in Eq. (21) may be obtained, we have the following lower bounds to the robustness measures for any state. For all $Q \subset \mathcal{Q}$, we have

$$
\begin{gathered}
\mathcal{E}_{R}[\hat{\varrho}] \geq \frac{\langle\hat{S}\rangle-N|Q| s}{N^{2} s^{2}}-1=: E_{R}[\hat{\varrho}], \\
\mathcal{E}_{G R}[\hat{\varrho}] \geq \frac{\langle\hat{S}\rangle}{N|Q| s+N^{2} s^{2}}-1=: E_{G R}[\hat{\varrho}] .
\end{gathered}
$$

We present $E_{R}[\hat{\varrho}]$ and $E_{G R}[\hat{\varrho}]$ for several numerically simulated states in Section III, see Fig. 4, and discuss some analytic examples in Section IV.

\section{NUMERICAL ANALYSIS OF MAGNETIC MATERIALS}

For all our numerical examples we assume that $f_{i, \alpha}(\boldsymbol{q})=$ $f(\boldsymbol{q})$, that the $N=L^{3}$ spins are arranged on a simple cubic lattice with $\boldsymbol{r}_{i}=\boldsymbol{i} \in\{1, \ldots, L\}^{\times 3}$ and periodic boundary conditions, and that

$$
Q \subset 2 \pi\{0, \ldots, L-1\}^{\times 3} / L
$$

We will consider ground and thermal states, $\hat{\varrho}=$ $\mathrm{e}^{-\hat{H} /\left(k_{B} T\right)} / Z$, of quasi-one- and two-dimensional Hamiltonians, that is, Hamiltonians of the form

$$
\hat{H}=\sum_{i_{z}, i_{y}=1}^{N} \hat{H}_{1 D}^{\left(i_{z}, i_{y}\right)} \text { or } \hat{H}=\sum_{i_{z}=1}^{N} \hat{H}_{2 D}^{\left(i_{z}\right)}
$$

i.e., Hamiltonians that correspond to $L^{2}$ mutually uncoupled chains or Hamiltonians that correspond to $L$ mutually uncoupled two-dimensional systems. We further assume that the individual chains are governed by the same one-dimensional Hamiltonian $\hat{H}_{1 D}$ and will give numerical examples for the one-dimensional Heisenberg model and the $X Y$-chain. Similarly, we assume that the individual two-dimensional systems are governed by the same $\hat{H}_{2 D}$ and provide numerical examples for it being the two-dimensional Heisenberg model.

Results for thermal states are obtained using the loop algorithm of the ALPS quantum Monte Carlo library [47]. For details on the simulation of effective one- and two-dimensional models and the symmetries of the models under consideration see Appendix B and C.

We start with quasi-one-dimensional models, the first of which is the antiferromagnetic one-dimensional Heisenberg model, i.e., the individual chains are governed by the Hamiltonian

$$
\hat{H}_{1 D}^{H}=J \sum_{\langle i, j\rangle} \hat{\boldsymbol{S}}_{i} \cdot \hat{\boldsymbol{S}}_{j}=J \sum_{\langle i, j\rangle} \sum_{\alpha} \hat{S}_{i}^{\alpha} \hat{S}_{j}^{\alpha},
$$




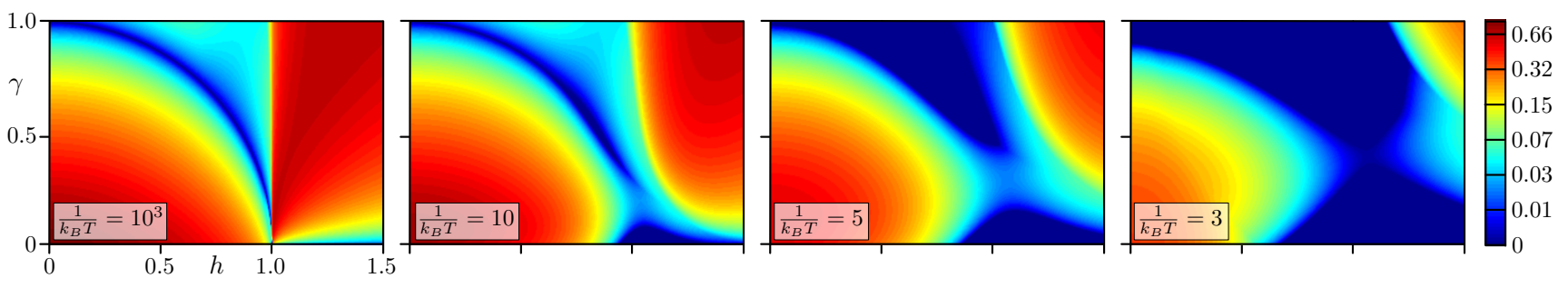

FIG. 2. Lower bound $E_{B S A}[\hat{\varrho}]$ (Eq. (20)) to the best separable approximation $\mathcal{E}_{B S A}[\hat{\varrho}]$ for thermal states of a system of mutually uncoupled chains, Eq. (26), each of which is described by the XY-model in Eq. (28). Linear dimension is $L=200$ and the depicted bounds are obtained by optimizing $E_{B S A}[\hat{\varrho}](\boldsymbol{q})$ over certain $\boldsymbol{q}$ and over the orientation of the chains (see main text). For low temperature, the phase boundary and factorization circle $\gamma^{2}+h^{2}=1$ are clearly visible. Note also that for higher temperature, there are regions on this circle with finite entanglement.

\begin{tabular}{|c|c|c|c|c|c|}
\hline Compound & Effective $D$ & $s$ & $J[\mathrm{~K}]$ & studied at $T[\mathrm{~K}]$ & in Ref. \\
\hline \hline $\mathrm{Cs}_{2} \mathrm{CuCl}_{4}$ & 1 & $1 / 2$ & 4 & 0.06 & {$[48]$} \\
\hline $\mathrm{CsNiCl}_{3}$ & 1 & 1 & 17 & 1.6 & {$[49]$} \\
\hline $\mathrm{CFTD}$ & 2 & $1 / 2$ & 72 & 1.5 & {$[50]$} \\
\hline $\mathrm{La}_{2} \mathrm{CuO}_{4}$ & 2 & $1 / 2$ & 1567 & 337 & {$[40]$} \\
\hline $\mathrm{SrCuO}_{4} \mathrm{Cl}_{2}$ & 2 & $1 / 2$ & 1451 & 10 & {$[41]$} \\
\hline $\mathrm{K}_{2} \mathrm{NiF}_{4}$ & 2 & 1 & 112 & 4.2 & {$[39]$} \\
\hline $\mathrm{Rb}_{2} \mathrm{MnF}_{4}$ & 2 & $5 / 2$ & 8 & 10 & {$[51]$} \\
\hline
\end{tabular}

Further, we maximize over three possible orientations of the chains in real space (oriented along the $x, y$, or $z$ direction), see Appendix C for details.

Finally, in Fig. 3, we present results for the quasi-two dimensional model, in which each two-dimensional subsystem is governed by the Heisenberg model such that the total Hamiltonian reads

$$
\hat{H}^{H}=J \sum_{\langle\boldsymbol{i}, \boldsymbol{j}\rangle} \delta_{i_{z}, j_{z}} \sum_{\alpha} \hat{S}_{\boldsymbol{i}}^{\alpha} \hat{S}_{\boldsymbol{j}}^{\alpha}
$$

TABLE I. Various materials that have been studied using neutron scattering and that may approximately be described by quasi-one- or two-dimensional Heisenberg Hamiltonians.

where $\langle\cdot, \cdot\rangle$ denotes summation over nearest neighbors. Various materials may approximately be described by such mutually uncoupled chains and have been studied experimentally using neutron scattering, see Table 1 for some examples. In Fig. 1, we present results for the lower bound $E_{B S A}[\hat{\varrho}](\boldsymbol{q})$, which, due to symmetries of the considered model, is independent of $q_{y}$ and $q_{z}$ (see Appendix C for details).

As a second quasi-one-dimensional example, we consider the spin- $1 / 2 \mathrm{XY}$-chain in a transverse magnetic field,

$$
\hat{H}_{1 D}^{X Y}=\sum_{\langle i, j\rangle}\left[(1+\gamma) \hat{S}_{i}^{x} \hat{S}_{j}^{x}+(1-\gamma) \hat{S}_{i}^{y} \hat{S}_{j}^{y}\right]-h \sum_{i} \hat{S}_{i}^{z}
$$

where $\gamma$ is the anisotropy parameter and $h$ denotes the magnetic field. The system undergoes a quantum phase transition at the critical value $h=1$ and the ground state factorizes for $\gamma^{2}+h^{2}=1$. See Ref. [52] for a comparison of this model to experimental data on $\mathrm{Cs}_{2} \mathrm{CoCl}_{4}$ and Ref. [53] for confirmation of the one-dimensional spin- $1 / 2 \mathrm{XY}$ character of the interactions between the pseudospins of the $\mathrm{Pr}^{3+}$ ions in $\mathrm{PrCl}_{3}$. The spin-correlation functions for thermal states of this model were extensively studied by Barouch and McCoy in [54] and may be obtained numerically for very large chain lengths. We present lower bounds to the best separable approximation of thermal states of this model in Fig. 2. These bounds are obtained by maximizing the bound $E_{B S A}[\hat{\varrho}](\boldsymbol{q})$ over all $\boldsymbol{q} \in 2 \pi\{0, \ldots, L-1\}^{\times 3} / L$ with $\boldsymbol{q} \neq \mathbf{0}$ and $\bar{q}_{x} \bar{q}_{y}=0$.

where we recall that $i=\left(i_{x}, i_{y}, i_{z}\right) \in\{1, \ldots, L\}^{\times 3}$. Due to the symmetries of this model, the bound $E_{B S A}[\hat{\varrho}](\boldsymbol{q})$ in Eq. (20) is independent of $q_{z}$, see Appendix $C$ for details. For compounds well described by the quasi-two-dimensional Heisenberg model see Table 1.

To present results on the robustness measures $\mathcal{E}_{R}[\hat{\varrho}]$ and $\mathcal{E}_{G R}[\hat{\varrho}]$, we need to specify the set of scattering vectors $Q$ appearing in the lower bounds $E_{R}[\hat{\varrho}]$ and $E_{G R}[\hat{\varrho}]$ in Eqs. $(24,21)$. We use the following choice of scattering vectors:

$$
Q(x)=\left\{\boldsymbol{q} \in \mathcal{Q} \mid \sum_{i, j} \mathrm{e}^{\mathrm{i} \boldsymbol{q}\left(\boldsymbol{r}_{i}-\boldsymbol{r}_{j}\right)} \sum_{\alpha}\left\langle\hat{S}_{i}^{\alpha} \hat{S}_{j}^{\alpha}\right\rangle \geq x\right\}
$$

and then take $Q$ as the $Q(x)$ that maximizes the lower bound. In Fig. 4, we present results for all the Heisenberg models that we also considered for the best separable approximation.

\section{ANALYTIC EXAMPLES}

In this section, we discuss resonating valence bond (RVB) states and the Majumdar-Gosh model, for which an exact expression for the expectation value of $\hat{S}(\boldsymbol{q})$ (and hence for our entanglement bounds) may be obtained.

In the context of high temperature superconductors, resonating valence bond (RVB) states were introduced by Anderson $[55,56]$. They are used to describe quantum-spin-liquids, i.e., states without long-range magnetic order [57], and appear as ground states of frustrated antiferromagnets. Such systems and their description by RVB currently receive increased theoretical as well as experimental attention, see, e.g., 

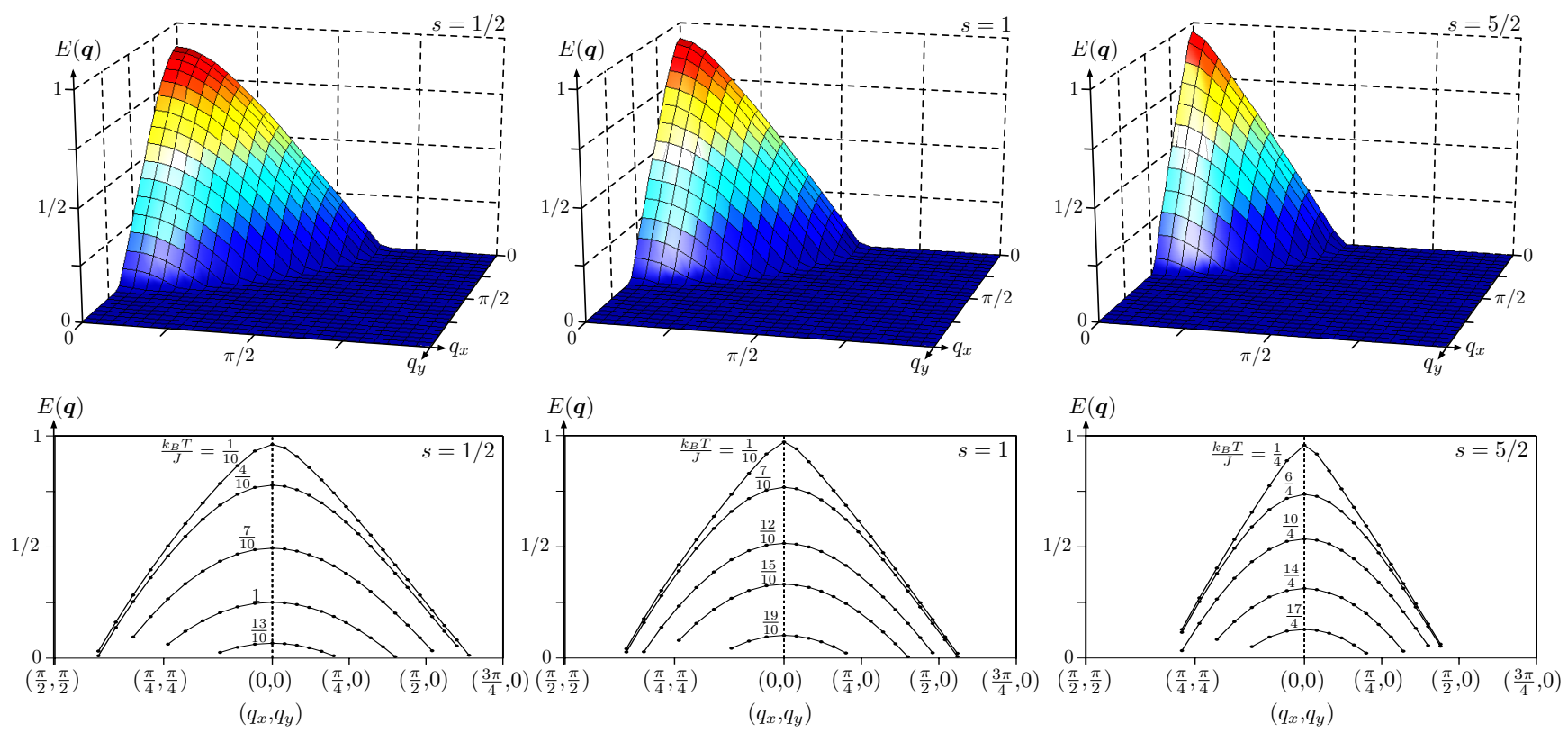

FIG. 3. Lower bound $E_{B S A}[\hat{\varrho}](\boldsymbol{q})=E_{B S A}[\hat{\varrho}]\left(q_{x}, q_{y}\right)$ (Eq. (20)) to the entanglement $\mathcal{E}_{B S A}[\hat{\varrho}]$ for thermal states of the quasi-two-dimensional Heisenberg model in Eq. (29) with $s=1 / 2,1,5 / 2$ (left to right). Top row shows $E_{B S A}[\varrho]\left(q_{x}, q_{y}\right)$ for $T / J=1 / 4$, bottom row shows cuts through the first Brillouin zone for different temperatures. Cuts are along the line from $\left(q_{x}, q_{y}\right)=(\pi / 2, \pi / 2)$ to $(0,0)$ and and along the $x$-axis from $(0,0)$ to $(3 \pi / 4,0)$. Simulated system size is $L=30$ [68]. Lines are guides to the eye and only data points with $E_{B S A}[\hat{\varrho}]\left(q_{x}, q_{y}\right)>0$ and $\left(q_{x}, q_{y}\right) \in 2 \pi\{0, \ldots, L-1\}^{\times 2} / L$ are shown.

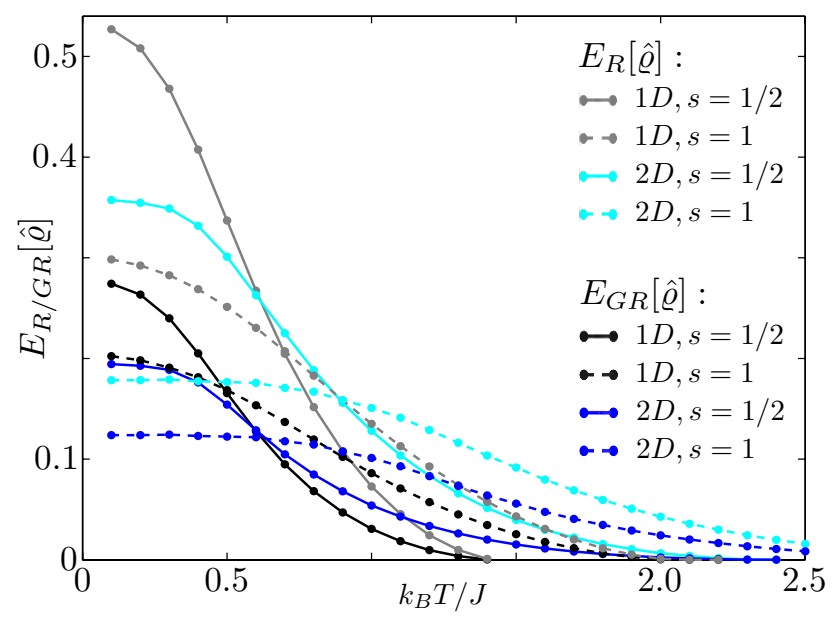

FIG. 4. Lower bounds $E_{R}[\hat{\varrho}]$ and $E_{G R}[\hat{\varrho}]$ (Eqs. (24,21), see main text for the choice of the set $Q$ ) to the robustness measures $\mathcal{E}_{R}[\hat{\varrho}]$ and $\mathcal{E}_{G R}[\hat{\varrho}]$ as a function of temperature for thermal states of the quasi-one-dimensional (black, see Eqs. $(26,27)$ ) and quasi-twodimensional (blue, see Eq. (27)) Heisenberg model with spin number $s=1 / 2$ (solid) and $s=1$ (dashed). Note that for any state $E_{R}[\hat{\varrho}] \geq E_{G R}[\hat{\varrho}]$. Lines are guides to the eye and $N=900$ spins were simulated [68].

Refs. [8, 9, 58, 59] and Ref. [60] for a recent neutron scattering investigation of the antiferromagnetic Heisenberg model on a Kagomé lattice. Besides the characterization of high- $T_{c}$ superconductors, quantum spin liquids have potential applications for topological quantum computation [61]. The entanglement properties of RVB states have recently been considered using tools from quantum information theory [62, 63]. Consider a lattice with $N$ (even) sites and a dimer covering $\Delta=\left\{\left(i_{1}, j_{1}\right), \ldots,\left(i_{N / 2}, j_{N / 2}\right)\right\}$, i.e., a collection of pairs of lattice sites such that each lattice site belongs to exactly one dimer. To any such dimerization, one may associate a valence bond state $\left|\psi_{\Delta}\right\rangle=\otimes_{(i, j) \in \Delta}\left|\phi_{i, j}\right\rangle$. Singlet RVB states are superpositions of such states, $|\psi\rangle=\sum_{\Delta} c_{\Delta}\left|\psi_{\Delta}\right\rangle$, where each dimer forms a singlet, $\left|\phi_{i, j}\right\rangle=\frac{1}{\sqrt{2}}\left(|\uparrow\rangle_{i}|\downarrow\rangle_{j}-|\downarrow\rangle_{i}|\uparrow\rangle_{j}\right)$. The span of all singlet valence bond states is equal to the singlet sector, i.e., to the spin-zero subspace. For these states, in the limit $\boldsymbol{q} \rightarrow \mathbf{0}$, we have $E_{B S A}[|\psi\rangle\langle\psi|](\boldsymbol{q} \rightarrow \mathbf{0}) \geq$ $1-\frac{1}{N C_{s}} \sum_{\alpha}\left\langle\hat{S}_{\alpha}^{2}\right\rangle=1$, where $\hat{S}_{\alpha}=\sum_{i} \hat{S}_{i}^{\alpha}$ the total spin along $\alpha$, i.e.,

$$
E_{B S A}[|\psi\rangle\langle\psi|](\boldsymbol{q} \rightarrow \mathbf{0})=1
$$

for all $|\psi\rangle=\sum_{\Delta} c_{\Delta}\left|\psi_{\Delta}\right\rangle$, i.e., these states maximally violate the lower bound in Eq. (14) and their entanglement as quantified in terms of the best separable approximation is hence optimally quantified by the neutron scattering observable in Eq. (20).

Hamiltonians for which RVB may describe the ground state and explain low-lying excitations include examples with frustration due to additional next-nearest-neighbor interaction such as the so called Klein Hamiltonian [64] on twodimensional lattices and the Majumdar-Ghosh Hamiltonian in 
one dimension [65],

$$
\hat{H}_{1 D}^{M G}=2 \sum_{i} \hat{\boldsymbol{S}}_{i} \cdot \hat{\boldsymbol{S}}_{i+1}+\sum_{i} \hat{\boldsymbol{S}}_{i} \cdot \hat{\boldsymbol{S}}_{i+2}
$$

In Ref. [66] it was shown that the ratio of nearest-neighbor and next-nearest neighbor coupling in the quasi-one-dimensional antiferromagnet $\mathrm{CuCrO}_{4}$ is close to 2, putting this magnet in the vicinity of the Majumdar-Ghosh point. Every ground state of $\hat{H}_{1 D}^{M G}$ is a superposition of two two-periodic states given by products of nearest-neighbor singlets, i.e., a RVB. The equal weight ground state may be given explicitly exploiting its description as a matrix product state [67]. The correlators can be computed exactly and allow for a particularly concise expression of the structure factor in the thermodynamic limit: The correlators for a single chain of length $L$ are given by

$$
\left\langle\hat{S}_{i}^{\alpha} \hat{S}_{i+r}^{\alpha}\right\rangle= \begin{cases}(-1)^{\frac{L}{2}-1} \frac{(-1)^{r}}{2^{\frac{L}{2}+1}+4(-1)^{\frac{L}{2}}} & \text { for } r>1 \\ -\frac{1}{4} \frac{2^{\frac{L}{2}}+4(-1)^{\frac{L}{2}}}{2^{\frac{L}{2}+1}+4(-1)^{\frac{L}{2}}} & \text { for } r=1\end{cases}
$$

In the thermodynamic limit we find $\left\langle\hat{S}_{i}^{\alpha} \hat{S}_{i+r}^{\alpha}\right\rangle=-\frac{\delta_{1, r}}{8}$ for $\alpha=x, y, z$ and $r>0$, which yields

$$
E_{B S A}[\hat{\varrho}](\boldsymbol{q})=2 \cos \left(q_{x}\right)-1
$$

if every one of the mutually uncoupled chains is in this ground state, see solid line in Fig. 1 . With $Q=\{\boldsymbol{q}\}$, i.e., $|Q|=1$, we find for the robustness bounds in Eq. (24) that, as $N \rightarrow \infty$,

$$
\begin{gathered}
E_{R}[\hat{\varrho}]=\frac{1-3 \cos \left(q_{x}\right)}{N}-1, \\
E_{G R}[\hat{\varrho}]=\frac{3\left(1-\cos \left(q_{x}\right)\right)}{2+N}-1,
\end{gathered}
$$

both of which become trivial if $N$ is too large. Just as for the numerical examples, we see that summation over several scattering vectors is necessary to obtain a non-trivial lower bound: We choose $Q=\left\{\boldsymbol{q} \in \mathcal{Q} \mid \frac{2 \pi}{L} \frac{L}{2}-\frac{2 \pi}{L} c L<q_{x} \leq\right.$ $\left.\frac{2 \pi}{L} \frac{L}{2}+\frac{2 \pi}{L} c L\right\}$, i.e., $|Q|=2 c N$. We may then, for large $L$, replace the summation of the structure factor over different $q_{x}$ by an integral according to $\lim _{L \rightarrow \infty} \frac{1}{L} \sum_{q=a}^{b} f\left(\frac{2 \pi q}{L}\right)=$ $\frac{1}{2 \pi} \int_{2 \pi a / L}^{2 \pi b / L} f(q) \mathrm{d} q$. By direct computation of the integral and then maximizing over $0<c<1 / 2$, we find that $E_{R}[\hat{\varrho}] \approx$ 0.51 and $E_{G R}[\hat{\varrho}] \approx 0.23$ in the thermodynamic limit.

\section{SUMMARY AND OUTLOOK}

We showed how entanglement may be quantified relying on observables typically obtained in scattering experiments. In particular, these observables can be measured via the scattering cross-section in neutron scattering. We showed how such measurements give lower bounds on the entanglement in the sample, bounding the best separable approximation, the robustness of entanglement and the generalized robustness of entanglement. These bounds do neither rely on the knowledge of the systems underlying Hamiltonian nor any other information about the state of the sample material. The detection can be applied to macroscopic systems, because the experimental effort does not increase with the system size - in stark contrast to quantum state tomography. We showed for several model Hamiltonians such as the Heisenberg, MajumdarGosh and XY models (for different spin numbers and different spatial geometries), that our method can indeed quantify entanglement in large samples at finite temperature. Interestingly, quantum phase transitions and factorization points are detected by our entanglement bounds. The considered models are well known and applicable to real materials. Therefore our results pave the way for macroscopic entanglement quantification in experiments. This is very important for future applications, which utilize entanglement, e.g. in quantum information science. Our method might also be valuable as an alternative way to check the power of a model to describe the sample material, e.g., if a sample is highly entangled, a classical description certainly fails.

\section{ACKNOWLEDGMENTS}

We gratefully acknowledge Robert Rosenbach for help with the numerics. The work at Ulm University has been supported by the EU Integrated Projects Q-ESSENCE and SIQS, the EU STREP EQUAM, the BMBF Verbundprojekt QuOReP, a GIF project, and an Alexander von Humboldt Professorship. D.B., M.E. and H.K. acknowledge financial support by the Deutsche Forschungsgemeinschaft (DFG).
[1] M.B. Plenio and S. Virmani, Quant. Inf. Comp. 7, 1 (2007).

[2] R. Horodecki, P. Horodecki, M. Horodecki, and K. Horodecki, Rev. Mod. Phys. 81, 865 (2009).

[3] K. Audenaert, J. Eisert, M.B. Plenio, and R.F. Werner, Phys. Rev. A 66, 042327 (2002).

[4] T.J. Osborne and M.A. Nielsen, Quant. Inf. Comp. 1, 45 (2002).

[5] G. Vidal, Phys. Rev. Lett. 91, 147902 (2003).

[6] M.B. Plenio, J. Eisert, J. Dreissig, and M. Cramer, Phys. Rev. Lett. 94, 060503 (2005).
[7] J. Eisert, M. Cramer, and M.B. Plenio, Rev. Mod. Phys. 82, 277 (2010).

[8] H.-C. Jiang, Z. Wang, and L. Balents, Nat. Phys. 8, 902 (2012).

[9] S. Depenbrock, I.P. McCulloch, and U. Schollwoeck, Phys. Rev. Lett. 109, 067201 (2012).

[10] O. Gühne, P. Hyllus, D. Bruß, A. Ekert, M. Lewenstein, C. Macchiavello, and A. Sanpera, Phys. Rev. A 66, 062305 (2002).

[11] O. Gühne and G. Toth, Physics Reports 474, 1 (2009).

[12] M. Barbieri, F. De Martini, G. Di Nepi, P. Mataloni, G. M. D'Ariano, and C. Macchiavello, Phys. Rev. Lett. 91, 227901 
(2003)

[13] M. Bourennane, M. Eibl, C. Kurtsiefer, S. Gaertner, H. Weinfurter, O. Gühne, P. Hyllus, D. Bruß, M. Lewenstein, and A. Sanpera, Phys. Rev. Lett. 92, 087902 (2004).

[14] D. Leibfried, E. Knill, S. Seidelin, J. Britton, R.B. Blakestad, J. Chiaverini, D.B. Hume, W.M. Itano, J.D. Jost, C. Langer, R. Ozeri, R. Reichle, and D.J. Wineland, Nature 438, 639 (2005).

[15] H. Häffner, W. Hänsel, C.F. Roos, J. Benhelm, D. Chek-al-kar, M. Chwalla, T. Körber, U.D. Rapol, M. Riebe, P.O. Schmidt, C. Becher, O. Gühne, W. Dür, and R. Blatt, Nature 438, 643 (2005).

[16] J. Estève, C. Gross, A. Weller, S. Giovanazzi, and M.K. Oberthaler, Nature 455, 1216 (2008).

[17] I.D. Leroux, M.H. Schleier-Smith, and V. Vuletić, Phys. Rev. Lett. 104, 250801 (2010).

[18] M.F. Riedel, P. Böhi, Y. Li, T.W. Hänsch, A. Sinatra, and P. Treutlein, Nature 464, 1170 (2010).

[19] A. Louchet-Chauvet, J. Appel, J. J. Renema, D. Oblak, N. Kjaergaard and E. S. Polzik, New. Journal of Phys. 12, 065032 (2010).

[20] T. Monz, P. Schindler, J.T. Barreiro, M. Chwalla, D. Nigg, W.A. Coish, M. Harlander, W. Hänsel, M. Hennrich, and R. Blatt, Phys. Rev. Lett. 106, 130506 (2011).

[21] X.-C. Yao, T.-X. Wang, P. Xu, H. Lu, G.-S. Pan, X.-H. Bao, C.Z. Peng, C.-Y. Lu, Y.-A. Chen, and J.-W. Pan, Nat. Photonics 6, 225 (2012).

[22] A. Chiuri, G. Vallone, N. Bruno, C. Macchiavello, D. Bruß, and P. Mataloni, Phys. Rev. Lett. 105, 250501 (2010).

[23] M. Cramer, A. Bernard, N. Fabbri, L. Fallani, C. Fort, S. Rosi, F. Caruso, M. Inguscio, and M.B. Plenio, Nat. Commun. 4, 2161 (2013).

[24] H. Wunderlich, G. Vallone, P. Mataloni, and M.B. Plenio, New J. Phys. 13033033 (2011).

[25] K. Vogel and H. Risken, Phys. Rev. A 40, 2847 (1989).

[26] M. Cramer, M.B. Plenio, S.T. Flammia, R. Somma, D. Gross, S.D. Bartlett, O. Landon-Cardinal, D. Poulin, and Y.-K. Liu, Nat. Commun. 1, 149 (2010).

[27] P. Krammer, H. Kampermann, D. Bruß, R.A. Bertlmann, L.C. Kwek and C. Macchiavello, Phys. Rev. Lett. 103, 100502 (2009).

[28] M. Cramer, M.B. Plenio, and H. Wunderlich, Phys. Rev. Lett. 106, 020401 (2011).

[29] R. Horodecki, M. Horodecki and P. Horodecki, Phys. Rev. A 59, 1799 (1999).

[30] F.G.S.L. Brandão, Phys. Rev. A 72022310 (2005).

[31] K.M.R. Audenaert and M.B. Plenio, New J. Phys. 8, 266 (2006).

[32] O. Gühne, M. Reimpell, and R.F. Werner, Phys. Rev. Lett. 98, 110502 (2007).

[33] J. Eisert, F.G.S.L. Brandão and K.M.R. Audenaert,New J. Phys. 9, 46 (2007).

[34] T.A. Corcovilos, S.K. Baur, J.M. Hitchcock, E.J. Mueller, and R.G. Hulet, Phys. Rev. A 81, 013415 (2010).

[35] C. Macchiavello and G. Morigi, Phys. Rev. A 87, 044301 (2013).

[36] I.A. Zaliznyak and S.-H. Lee., Magnetic Neutron scattering, in Modern Techniques for Characterizing Magnetic Materials, edited by Y. Zhu (Springer, Heidelberg, 2005).

[37] L. Van Hove, Phys. Rev. 95, 249 (1954).

[38] M.P. Schulhof, P. Heller, R. Nathans, and A. Linz, Phys. Rev. B 1, 2304 (1970); E. Balcar and S.W. Lovesey, Theory of Mag- netic Neutron and Photon Scattering, Oxford University Press, Chapt. 1.7 (1989).

[39] R.J. Birgeneau, J. Skalyo, and G. Shirane, Phys. Rev. B 3, 1736 (1971).

[40] R.J. Birgeneau, M. Greven, M.A. Kastner, Y.S. Lee, B.O. Wells, Y. Endoh, K. Yamada, and G. Shirane, Phys. Rev. B 59, 13788 (1999).

[41] Y.J. Kim, R.J. Birgeneau, F.C. Chou, M. Greven, M.A. Kastner, Y.S. Lee, B.O. Wells, A. Aharony, O. Entin-Wohlman, I.Ya. Korenblit, A.B. Harris, R.W. Ewin, and G. Shirane, Phys. Rev. B 64, 024435 (2001).

[42] M. Lewenstein and A. Sanpera, Phys. Rev. Lett. 80, 2261 (1998).

[43] G. Vidal and R. Tarrach, Phys. Rev. A 59, 141 (1999).

[44] M. Steiner, Phys. Rev. A 67, 054305 (2003).

[45] H.F. Hofmann and S. Takeuchi, Phys. Rev. A 68, 032103 (2003).

[46] Q.Y. He, Shi-Guo Peng, P.D. Drummond, and M.D. Reid, Phys. Rev. A 84, 022107 (2011).

[47] A.F. Albuquerque et al., J. of Magn. and Magn. Materials 310, 1187 (2007); http://alps.comp-phys.org; For all numerical results we used 150000 Monte Carlo steps (after thermalization) ("sweeps") and 15000 sweeps for thermalization ("thermalization").

[48] R. Coldea, D.A. Tennant, R.A. Cowley, D. F. McMorrow, B. Dorner and Z. Tylczynski, Phys. Rev. Lett. 79, 151 (1997).

[49] M. Steiner, K. Kakurai, J.K. Kjems, D. Petitgrand and R. Pynn, J. Appl. Phys. 61, 3953 (1987).

[50] N.B. Christensen, H.M. Rønnow, D.F. McMorrow, A. Harrison, T.G. Perring, M. Enderle, R. Coldea, L.P. Regnault and G. Aeppli, PNAS 104, 15264 (2007)

[51] Y.S. Lee, M. Greven, B.O. Wells, R.J. Birgeneau and G. Shirane, Eur. Phys. J. B 5, 15 (1998)

[52] R. Basak and I. Chatterjee, Phys. Rev. B, 40, 4627 (1989).

[53] E. Goovaerts, H. DeRaedt and D. Schoemaker, Phys. Rev. Lett. 52, 1649 (1984).

[54] E. Barouch and B.M. McCoy, Phys. Rev. A 3, 786 (1971).

[55] P.W. Anderson, Mater. Res. Bull. 8, 153 (1973).

[56] P.W. Anderson, Science 235, 1196 (1987).

[57] L. Balents, Nature 464, 199 (2010).

[58] S. Yan, D.A. Huse, and S.R. White, Science 332, 1173 (2011).

[59] N. Schuch, D. Poilblanc, J.I. Cirac, and D. Perez-Garcia, Phys. Rev. B 86, 115108 (2012).

[60] T.-H. Han, J.S. Helton, S. Chu, D.G. Nocera, J.A. RodriguezRivera, C. Broholm, and Y.S. Lee, Nature 492, 406 (2012).

[61] A.Y. Kitaev, Ann. Phys. 303, 2 (2003).

[62] A. Chandran, D. Kaszlikowski, A. Sen(De), U. Sen, and V. Vedral, Phys. Rev. Lett. 99, 170502 (2007).

[63] D. Poilblanc, N. Schuch, D. Perez-Garcia, J.I. Cirac, Phys. Rev. B 86, 014404 (2012).

[64] J.D. Klein, J. Phys. A: Math. Gen. 15, 661 (1982).

[65] C.K. Majumdar, and D.K. Ghosh, J. Math. Phys. 101388 (1969).

[66] J.M. Law, P. Reuvekamp, R. Glaum, C. Lee, J. Kang, M.-H. Whangbo, and R.K. Kremer, arXiv:1106.0662.

[67] D. Perez-Garcia, F. Verstraete, M.M. Wolf, and J.I. Cirac, Quantum Inf. Comput. 7, 401 (2007).

[68] We have studied systems with total number of spins varying between 100 and 2500 and found no noticeable finite-size effects (differences between the obtained bounds were smaller than 0.01). 


\section{Appendix A: Bounds for fully separable states}

Let $Q \subset \mathbb{R}^{3}$. For $\boldsymbol{q} \in Q, i=1, \ldots, N$, and $\alpha=x, y, z$, let $f_{i, \alpha}(\boldsymbol{q}) \in \mathbb{C}$ and $\boldsymbol{r}_{i} \in \mathbb{R}^{3}$. Further, for each $\boldsymbol{q} \in Q$ let $M(\boldsymbol{q})$ be a $3 \times 3$ Hermitian positive semi-definite matrix with entries $M_{\alpha, \beta}(\boldsymbol{q})$. Consider the observable

$$
\hat{S}=\sum_{\boldsymbol{q} \in Q} \sum_{\alpha, \beta} \sum_{i, j} M_{\alpha, \beta}(\boldsymbol{q}) f_{i, \alpha}^{*}(\boldsymbol{q}) f_{j, \beta}(\boldsymbol{q}) \mathrm{e}^{\mathrm{i} \boldsymbol{q}\left(\boldsymbol{r}_{i}-\boldsymbol{r}_{j}\right)} \hat{S}_{i}^{\alpha} \hat{S}_{j}^{\beta},
$$

which is positive semi-definite: Denoting $\hat{S}_{\alpha}(\boldsymbol{q})=\sum_{i} f_{i, \alpha}(\boldsymbol{q}) \mathrm{e}^{-\mathrm{i} \boldsymbol{q} \boldsymbol{r}_{i}} \hat{S}_{i}^{\alpha}$, we have

$$
\hat{S}=\sum_{\boldsymbol{q} \in Q} \sum_{\alpha, \beta} M_{\alpha, \beta}(\boldsymbol{q}) \hat{S}_{\alpha}(\boldsymbol{q})^{\dagger} \hat{S}_{\beta}(\boldsymbol{q}),
$$

which is positive semidefinite as the $M_{\alpha, \beta}(\boldsymbol{q})$ are and as for every $\boldsymbol{q}$ and every state vector $|\psi\rangle$ the $3 \times 3$ matrix with entries $\left\langle\psi\left|\hat{S}_{\alpha}(\boldsymbol{q})^{\dagger} \hat{S}_{\beta}(\boldsymbol{q})\right| \psi\right\rangle$ is positive semidefinite.

For each $i=1, \ldots, N$ define the $3 \times 3$ matrix $M^{(i)}$ with entries

$$
M_{\alpha, \beta}^{(i)}=\sum_{\boldsymbol{q} \in Q} f_{i, \alpha}^{*}(\boldsymbol{q}) f_{i, \beta}(\boldsymbol{q}) M_{\alpha, \beta}(\boldsymbol{q}) .
$$

We assume that these matrices are real symmetric. This is fulfilled, e.g., if $f_{i, \alpha}(\boldsymbol{q})=F_{i}(\boldsymbol{q}) g_{i, \alpha}$ with $F_{i}(\boldsymbol{q}) \in \mathbb{C}$ and $g_{i, \alpha} \in \mathbb{R}$. We further note that these $M^{(i)}$ are positive semidefinite as we assumed that the $M_{\alpha, \beta}(\boldsymbol{q})$ are positive semidefinite.

We set out to derive upper and lower bounds on the expectation of $\hat{S}$ for product states $|\psi\rangle=\otimes_{i}\left|\psi_{i}\right\rangle$. The same bounds then also hold for fully separable states $\hat{\varrho}=\sum_{n} p_{n} \otimes_{i} \hat{\varrho}_{i}^{(n)}$ by convexity. For product states, we have that for all $i \neq j$ the equality $\left\langle\hat{S}_{i}^{\alpha} \hat{S}_{j}^{\beta}\right\rangle=\left\langle\hat{S}_{i}^{\alpha}\right\rangle\left\langle\hat{S}_{j}^{\beta}\right\rangle$ holds. Hence,

$$
\begin{aligned}
\langle\hat{S}\rangle= & \sum_{i} \sum_{\alpha, \beta} M_{\alpha, \beta}^{(i)}\left\langle\hat{S}_{i}^{\alpha} \hat{S}_{i}^{\beta}\right\rangle-\sum_{i} \sum_{\alpha, \beta} M_{\alpha, \beta}^{(i)}\left\langle\hat{S}_{i}^{\alpha}\right\rangle\left\langle\hat{S}_{i}^{\beta}\right\rangle \\
& +\sum_{\boldsymbol{q} \in Q} \sum_{\alpha, \beta} \sum_{i, j} M_{\alpha, \beta}(\boldsymbol{q}) f_{i, \alpha}^{*}(\boldsymbol{q}) f_{j, \beta}(\boldsymbol{q}) \mathrm{e}^{\mathrm{i} \boldsymbol{q}\left(\boldsymbol{r}_{i}-\boldsymbol{r}_{j}\right)}\left\langle\hat{S}_{i}^{\alpha}\right\rangle\left\langle\hat{S}_{j}^{\beta}\right\rangle \\
= & : A-B+C .
\end{aligned}
$$

\section{Lower bound}

The third term, $C$, in Eq. (A4) is non-negative as for each $\boldsymbol{q}$ the matrix $M(\boldsymbol{q})$ is positive semidefinite. Hence we have the lower bound

$$
\langle\hat{S}\rangle \geq A-B=\sum_{i} \sum_{\alpha, \beta} M_{\alpha, \beta}^{(i)}\left(\left\langle\hat{S}_{i}^{\alpha} \hat{S}_{i}^{\beta}\right\rangle-\left\langle\hat{S}_{i}^{\alpha}\right\rangle\left\langle\hat{S}_{i}^{\beta}\right\rangle\right)
$$

As we assumed that for each $i$ the $M^{(i)}$ are real symmetric, there are mutually orthonormal real eigenvectors $\boldsymbol{m}_{\gamma}^{(i)}$ with corresponding eigenvalues $m_{\gamma}^{(i)}$ and there is a unitary $\hat{U}_{i}$ such that $\sum_{\alpha}\left[\boldsymbol{m}_{\gamma}^{(i)}\right]_{\alpha} \hat{S}_{i}^{\alpha}=\hat{U}_{i}^{\dagger} \hat{S}_{i}^{\gamma} \hat{U}_{i}$ for all $\gamma$. Thus

$$
\begin{aligned}
\sum_{\alpha, \beta} M_{\alpha, \beta}^{(i)}\left(\left\langle\psi_{i}\left|\hat{S}_{i}^{\alpha} \hat{S}_{i}^{\beta}\right| \psi_{i}\right\rangle-\left\langle\psi_{i}\left|\hat{S}_{i}^{\alpha}\right| \psi_{i}\right\rangle\left\langle\psi_{i}\left|\hat{S}_{i}^{\beta}\right| \psi_{i}\right\rangle\right) & =\sum_{\gamma} m_{\gamma}^{(i)}\left(\left\langle\psi_{i}\left|\hat{U}_{i}^{\dagger}\left(\hat{S}_{i}^{\gamma}\right)^{2} \hat{U}_{i}\right| \psi_{i}\right\rangle-\left\langle\psi_{i}\left|\hat{U}_{i}^{\dagger} \hat{S}_{i}^{\gamma} \hat{U}_{i}\right| \psi_{i}\right\rangle^{2}\right) \\
& \geq \min _{\substack{|\psi| \psi \mid \\
\langle\psi| \psi \mathbb{C}^{2 s+1}}} \sum_{\gamma} m_{\gamma}^{(i)}\left(\left\langle\psi\left|\left(\hat{S}_{i}^{\gamma}\right)^{2}\right| \psi\right\rangle-\left\langle\psi\left|\hat{S}_{i}^{\gamma}\right| \psi\right\rangle^{2}\right),
\end{aligned}
$$

which is $c_{\min }$ presented in the main text.

\section{Upper bound}

We first bound, similar to above,

$$
A=\sum_{i} \sum_{\alpha, \beta} M_{\alpha, \beta}^{(i)}\left\langle\psi_{i}\left|\hat{S}_{i}^{\alpha} \hat{S}_{i}^{\beta}\right| \psi_{i}\right\rangle=\sum_{i} \sum_{\gamma} m_{\gamma}^{(i)}\left\langle\psi_{i}\left|\hat{U}_{i}^{\dagger}\left(\hat{S}_{i}^{\gamma}\right)^{2} \hat{U}_{i}\right| \psi_{i}\right\rangle \leq \sum_{i}\left\|\sum_{\gamma} m_{\gamma}^{(i)}\left(\hat{S}_{i}^{\gamma}\right)^{2}\right\|
$$


Now denote by $\mathcal{M}$ the Hermitian $3 N \times 3 N$ matrix with entries

$$
\mathcal{M}_{i, \alpha ; j, \beta}=\sum_{\boldsymbol{q} \in Q} M_{\alpha, \beta}(\boldsymbol{q}) f_{i, \alpha}^{*}(\boldsymbol{q}) f_{j, \beta}(\boldsymbol{q})\left(\mathrm{e}^{\mathrm{i} \boldsymbol{q}\left(\boldsymbol{r}_{i}-\boldsymbol{r}_{j}\right)}-\delta_{i, j}\right) .
$$

This matrix has $\operatorname{tr}[\mathcal{M}]=0$, i.e., its largest eigenvalue $\lambda_{\max }$ is non-negative and therefore

$$
C-B=\sum_{\alpha, \beta} \sum_{i, j} \mathcal{M}_{i, \alpha ; j, \beta}\left\langle\hat{S}_{i}^{\alpha}\right\rangle\left\langle\hat{S}_{j}^{\beta}\right\rangle \leq \lambda_{\max } N s^{2} .
$$

Hence, we have the bound

$$
A-B+C \leq \sum_{i}\left\|\sum_{\gamma} m_{\gamma}^{(i)}\left(\hat{S}_{i}^{\gamma}\right)^{2}\right\|+\lambda_{\max } N s^{2} .
$$

This constitutes our general result for $c_{\max }$. To compute it, one needs to find the maximum eigenvalue of the $3 N \times 3 N$ matrix $\mathcal{M}$ and, for each $i=1, \ldots, N$, the eigenvalues of the $3 \times 3$ matrix $M^{(i)}$. We now discuss a geometry for which this may be made more explicit.

Let the positions of the $i$ 'th spin be $\boldsymbol{r}_{i}=\boldsymbol{r}_{k, l}=\boldsymbol{R}_{k}+\boldsymbol{x}_{l}$, where $k=1, \ldots, N_{c}$ and $l=1, \ldots, n$ such that $N=n N_{c}$. Further we let the lattice sites $k=1, \ldots, N_{c}$, with $N_{c}=N_{1}^{c} N_{2}^{c} N_{3}^{c}$, be the sites of a finite Bravais lattice with primitive vectors $\boldsymbol{a}_{d}$, $d=1,2,3$, such that $\boldsymbol{R}_{k}=\sum_{d=1}^{3} k_{d} \boldsymbol{a}_{d}$ with $k_{d} \in\left\{1, \ldots, N_{d}^{c}\right\}$. Note that this is more general than in the main text as we allow for $n$ spins in each unit cell. We now assume that $f_{i, \alpha}(\boldsymbol{q})=f_{k, l, \alpha}(\boldsymbol{q})=f_{l, \alpha}(\boldsymbol{q})$, i.e., depends only on $l$. Further we assume that

$$
Q \subset\left\{\sum_{d=1}^{3} q_{d} \boldsymbol{b}_{d} \mid q_{d} \in \frac{1}{N_{d}^{c}}\left\{0, \ldots, N_{d}^{c}-1\right\}\right\}=: \mathcal{Q},
$$

where the $\boldsymbol{b}_{d}$ are reciprocal primitive vectors corresponding to the $\boldsymbol{a}_{d}$. We then have $\frac{1}{N_{c}} \sum_{\boldsymbol{p} \in \mathcal{Q}} \mathrm{e}^{\mathrm{i} \boldsymbol{p}\left(\boldsymbol{R}_{k}-\boldsymbol{R}_{k^{\prime}}\right)}=\delta_{k, k^{\prime}}$, which yields

$$
\begin{aligned}
\mathcal{M}_{\alpha, k, l ; \beta, k^{\prime}, l^{\prime}}= & \sum_{\boldsymbol{q} \in Q} M_{\alpha, \beta}(\boldsymbol{q}) f_{l, \alpha}^{*}(\boldsymbol{q}) f_{l^{\prime}, \beta}(\boldsymbol{q})\left(\mathrm{e}^{\mathrm{i} \boldsymbol{q}\left(\boldsymbol{R}_{k}-\boldsymbol{R}_{k^{\prime}}\right)} \mathrm{e}^{\mathrm{i} \boldsymbol{q}\left(\boldsymbol{x}_{l}-\boldsymbol{x}_{l^{\prime}}\right)}-\delta_{k, k^{\prime}} \delta_{l, l^{\prime}}\right) \\
= & : \sum_{\boldsymbol{q} \in Q} M_{\alpha, l ; \beta, l^{\prime}}^{\prime}(\boldsymbol{q})\left(\mathrm{e}^{\mathrm{i} \boldsymbol{q}\left(\boldsymbol{R}_{k}-\boldsymbol{R}_{k^{\prime}}\right)}-\delta_{k, k^{\prime}} \delta_{l, l^{\prime}}\right) \\
= & \sum_{\boldsymbol{q} \in Q} M_{\alpha, l ; \beta, l^{\prime}}^{\prime}(\boldsymbol{q})\left(\mathrm{e}^{\mathrm{i} \boldsymbol{q}\left(\boldsymbol{R}_{k}-\boldsymbol{R}_{k^{\prime}}\right)}-\delta_{l, l^{\prime}} \frac{1}{N_{c}} \sum_{\boldsymbol{p} \in \mathcal{Q}} \mathrm{e}^{\mathrm{i} \boldsymbol{p}\left(\boldsymbol{R}_{k}-\boldsymbol{R}_{k^{\prime}}\right)}\right) \\
= & \sum_{\boldsymbol{q} \in Q} M_{\alpha, l ; \beta, l^{\prime}}^{\prime}(\boldsymbol{q}) \sum_{\boldsymbol{p} \in \mathcal{Q}}\left(\delta_{\boldsymbol{p}, \boldsymbol{q}}-\delta_{l, l^{\prime}} \frac{1}{N_{c}}\right) \mathrm{e}^{\mathrm{i} \boldsymbol{p}\left(\boldsymbol{R}_{k}-\boldsymbol{R}_{k^{\prime}}\right)} \\
= & : \sum_{\boldsymbol{p} \in \mathcal{Q}} M_{\alpha, l ; \beta, l^{\prime}}^{\prime \prime}(\boldsymbol{p}) \mathrm{e}^{\mathrm{i} \boldsymbol{p}\left(\boldsymbol{R}_{k}-\boldsymbol{R}_{k^{\prime}}\right)} \\
= & : \sum_{\boldsymbol{p} \in \mathcal{Q}}\left[M^{\prime \prime}(\boldsymbol{p}) \otimes \boldsymbol{e}_{\boldsymbol{p}} \boldsymbol{e}_{\boldsymbol{p}}^{\dagger}\right]_{\alpha, l, k ; \beta, l^{\prime}, k^{\prime}}
\end{aligned}
$$

and thus $\lambda_{\max }=N_{c} \max _{\boldsymbol{p} \in \mathcal{Q}} \lambda_{\max }\left[M^{\prime \prime}(\boldsymbol{p})\right]=N_{c} \max _{\boldsymbol{p} \in Q} \lambda_{\max }\left[M^{\prime \prime}(\boldsymbol{p})\right]$, where

$$
M_{\alpha, l ; \beta, l^{\prime}}^{\prime \prime}(\boldsymbol{p})=\sum_{\boldsymbol{q} \in Q} M_{\alpha, \beta}(\boldsymbol{q}) f_{l, \alpha}^{*}(\boldsymbol{q}) f_{l^{\prime}, \beta}(\boldsymbol{q}) \mathrm{e}^{\mathrm{i} \boldsymbol{q}\left(\boldsymbol{x}_{l}-\boldsymbol{x}_{l^{\prime}}\right)}\left(\delta_{\boldsymbol{p}, \boldsymbol{q}}-\delta_{l, l^{\prime}} \frac{1}{N_{c}}\right)
$$

Further,

$$
M_{\alpha, \beta}^{(i)}=M_{\alpha, \beta}^{(k, l)}=M_{\alpha, \beta}^{(l)}=\sum_{\boldsymbol{q} \in Q} f_{l, \alpha}^{*}(\boldsymbol{q}) f_{l, \beta}(\boldsymbol{q}) M_{\alpha, \beta}(\boldsymbol{q})
$$

with eigenvalues $m_{\gamma}^{(l)}$. We hence have the bound

$$
A-B+C \leq N_{c} \sum_{l=1}^{n}\left\|\sum_{\gamma} m_{\gamma}^{(l)}\left(\hat{S}_{i}^{\gamma}\right)^{2}\right\|+N s^{2} N_{c} \max _{\boldsymbol{p} \in Q} \lambda_{\max }\left[M^{\prime \prime}(\boldsymbol{p})\right]
$$


Comparing this $c_{\max }$ to the general bound above, one now has to, for each $\boldsymbol{q} \in Q$, find the maximum eigenvalue of a $3 n \times 3 n$ matrix (where we recall that $n$ is the number of spins in each unit cell) and, for each $l=1, \ldots, n$, find the eigenvalues of the $3 \times 3$ matrix $M^{(l)}$.

If $f_{l}(\boldsymbol{q})=f(\boldsymbol{q})$ and $M_{\alpha, \beta}(\boldsymbol{q})=\delta_{\alpha, \beta} /|f(\boldsymbol{q})|^{2}$, we have

$$
M_{\alpha, l ; \beta, l^{\prime}}^{\prime \prime}(\boldsymbol{p})=\delta_{\alpha, \beta} \sum_{\boldsymbol{q} \in Q} \mathrm{e}^{\mathrm{i} \boldsymbol{q}\left(\boldsymbol{x}_{l}-\boldsymbol{x}_{l^{\prime}}\right)}\left(\delta_{\boldsymbol{p}, \boldsymbol{q}}-\delta_{l, l^{\prime}} \frac{1}{N_{c}}\right)=\delta_{\alpha, \beta} \mathrm{e}^{\mathrm{i} \boldsymbol{p}\left(\boldsymbol{x}_{l}-\boldsymbol{x}_{l^{\prime}}\right)}-\delta_{\alpha, \beta} \delta_{l, l^{\prime}} \frac{|Q|}{N_{c}}
$$

and $M_{\alpha, \beta}^{(l)}=|Q| \delta_{\alpha, \beta}$, i.e., the bound simplifies to

$$
A-B+C \leq N|Q| s+N^{2} s^{2}
$$

which is $c_{\max }$ in the main text.

\section{Appendix B: Simulation details: Effective one- and two-dimensional systems}

Consider

$$
\hat{S}_{\alpha, \beta}(\boldsymbol{q})=\sum_{\boldsymbol{i}, \boldsymbol{j}} \mathrm{e}^{\mathrm{i} \boldsymbol{q}(\boldsymbol{i}-\boldsymbol{j})} \hat{S}_{\boldsymbol{i}}^{\alpha} \hat{S}_{\boldsymbol{j}}^{\beta}
$$

We write $\boldsymbol{i}=\left(i_{x} i_{y} i_{z}\right) \in\{1, \ldots, L\}^{\times 3}, \boldsymbol{q}=\left(q_{x} q_{y} q_{z}\right), \tilde{\boldsymbol{i}}=\left(i_{x} i_{y}\right), \tilde{\boldsymbol{q}}=\left(q_{x} q_{y}\right)$. If the system consists of mutually uncoupled (in the $z$-direction) two-dimensional systems, we have $\left\langle\hat{S}_{\boldsymbol{i}}^{\alpha} \hat{S}_{\boldsymbol{j}}^{\beta}\right\rangle=\left\langle\hat{S}_{\boldsymbol{i}}^{\alpha}\right\rangle\left\langle\hat{S}_{\boldsymbol{j}}^{\beta}\right\rangle$ whenever $i_{z} \neq j_{z}$, i.e.,

$$
\begin{aligned}
\left\langle\hat{S}_{\alpha, \beta}(\boldsymbol{q})\right\rangle & =\sum_{\substack{\boldsymbol{i}, \boldsymbol{j} \\
i_{z}=j_{z}}} \mathrm{e}^{\mathrm{i} \tilde{\boldsymbol{q}} \cdot(\tilde{\boldsymbol{i}}-\tilde{\boldsymbol{j}})}\left\langle\hat{S}_{\boldsymbol{i}}^{\alpha} \hat{S}_{\boldsymbol{j}}^{\beta}\right\rangle+\sum_{\substack{\boldsymbol{i}, \boldsymbol{j} \\
i_{z} \neq j_{z}}} \mathrm{e}^{\mathrm{i} \boldsymbol{q} \cdot(\boldsymbol{i}-\boldsymbol{j})}\left\langle\hat{S}_{\boldsymbol{i}}^{\alpha}\right\rangle\left\langle\hat{S}_{\boldsymbol{j}}^{\beta}\right\rangle \\
& =\sum_{\substack{\boldsymbol{i}, \boldsymbol{j} \\
i_{z}=j_{z}}} \mathrm{e}^{\mathrm{i} \tilde{\boldsymbol{q}} \cdot(\tilde{\boldsymbol{i}}-\tilde{\boldsymbol{j}})}\left(\left\langle\hat{S}_{\boldsymbol{i}}^{\alpha} \hat{S}_{\boldsymbol{j}}^{\beta}\right\rangle-\left\langle\hat{S}_{\boldsymbol{i}}^{\alpha}\right\rangle\left\langle\hat{S}_{\boldsymbol{j}}^{\beta}\right\rangle\right)+\left(\sum_{\boldsymbol{i}} \mathrm{e}^{\mathrm{i} \boldsymbol{q} \cdot \boldsymbol{i}}\left\langle\hat{S}_{\boldsymbol{i}}^{\alpha}\right\rangle\right)\left(\sum_{\boldsymbol{i}} \mathrm{e}^{\mathrm{i} \boldsymbol{q} \cdot \boldsymbol{i}}\left\langle\hat{S}_{\boldsymbol{i}}^{\beta}\right\rangle\right)^{*} \\
& =: S_{\alpha, \beta}(\tilde{\boldsymbol{q}})+M_{\alpha}(\boldsymbol{q}) M_{\beta}^{*}(\boldsymbol{q}) .
\end{aligned}
$$

Now let the two-dimensional sub-systems be equal. Then, any thermal state of the system is of the form $\hat{\varrho}=\otimes_{i_{z}} \hat{\varrho}_{i_{z}}$, where the $\hat{\varrho}_{i_{z}}$ are equal and each describes a two-dimensional layer at $z$ coordinate $i_{z}$. Hence,

$$
\left\langle\hat{S}_{\boldsymbol{i}}^{\alpha}\right\rangle=\operatorname{tr}\left[\hat{S}_{\boldsymbol{i}}^{\alpha} \hat{\varrho}\right]=\operatorname{tr}\left[\hat{S}_{\boldsymbol{i}}^{\alpha} \hat{\varrho}_{i_{z}}\right]=:\left\langle\hat{S}_{\tilde{\boldsymbol{i}}}^{\alpha}\right\rangle_{2 D}
$$

which does not depend on $i_{z}$. Similarly, for $i_{z}=j_{z}$,

$$
\left\langle\hat{S}_{\boldsymbol{i}}^{\alpha} \hat{S}_{\boldsymbol{j}}^{\beta}\right\rangle=\operatorname{tr}\left[\hat{S}_{\boldsymbol{i}}^{\alpha} \hat{S}_{\boldsymbol{j}}^{\beta} \hat{\varrho}\right]=\operatorname{tr}\left[\hat{S}_{\boldsymbol{i}}^{\alpha} \hat{S}_{\boldsymbol{j}}^{\beta} \hat{\varrho}_{i_{z}}\right]=:\left\langle\hat{S}_{\tilde{\boldsymbol{i}}}^{\alpha} \hat{S}_{\tilde{\boldsymbol{j}}}^{\beta}\right\rangle_{2 D},
$$

which does not depend on $i_{z}$. Hence,

$$
\frac{S_{\alpha, \beta}(\tilde{\boldsymbol{q}})}{L}=\sum_{\substack{i_{x}, i_{y}, j_{x}, j_{y}}} \mathrm{e}^{\mathrm{i} \tilde{\boldsymbol{q}} \cdot(\tilde{\boldsymbol{i}}-\tilde{\boldsymbol{j}})}\left(\left\langle\hat{S}_{\tilde{\boldsymbol{i}}}^{\alpha} \hat{S}_{\tilde{\boldsymbol{j}}}^{\beta}\right\rangle_{2 D}-\left\langle\hat{S}_{\tilde{\boldsymbol{i}}}^{\alpha}\right\rangle_{2 D}\left\langle\hat{S}_{\tilde{\boldsymbol{j}}}^{\beta}\right\rangle_{2 D}\right),
$$

which does not depend on $q_{z}$, and

$$
M_{\alpha}(\boldsymbol{q})=\sum_{\tilde{\boldsymbol{i}}} \mathrm{e}^{\mathrm{i} \tilde{\boldsymbol{q}} \tilde{\boldsymbol{i}}}\left\langle\hat{S}_{\tilde{\boldsymbol{i}}}^{\alpha}\right\rangle_{2 D} \sum_{i_{z}} \mathrm{e}^{\mathrm{i} q_{z} z_{i}}=L \delta_{q_{z}, 0} \sum_{\tilde{\boldsymbol{i}}} \mathrm{e}^{\mathrm{i} \tilde{\boldsymbol{q}} \cdot \tilde{\boldsymbol{i}}}\left\langle\hat{S}_{\tilde{\boldsymbol{i}}}^{\alpha}\right\rangle_{2 D},
$$

where we used that $q_{z} \in 2 \pi\{0, \ldots, L\} / L$. Similarly, if the system is quasi-one-dimensional with $\hat{\varrho}=\otimes_{i_{z}, i_{y}} \hat{\varrho}_{i_{z}, i_{y}}$ and all the $\hat{\varrho}_{i_{z}, i_{y}}$ equal, we have

$$
\left\langle\hat{S}_{\alpha, \beta}(\boldsymbol{q})\right\rangle=S_{\alpha, \beta}\left(q_{x}\right)+M_{\alpha}(\boldsymbol{q}) M_{\beta}(\boldsymbol{q})^{*},
$$

where,

$$
\begin{aligned}
\frac{S_{\alpha, \beta}(q)}{L^{2}} & =\sum_{i_{x}, j_{x}} \mathrm{e}^{\mathrm{i} q\left(i_{x}-j_{x}\right)}\left(\left\langle\hat{S}_{i_{x}}^{\alpha} \hat{S}_{j_{x}}^{\beta}\right\rangle_{1 D}-\left\langle\hat{S}_{i_{x}}^{\alpha}\right\rangle_{1 D}\left\langle\hat{S}_{j_{x}}^{\beta}\right\rangle_{1 D}\right), \\
\frac{M_{\alpha}(\boldsymbol{q})}{L^{2}} & =\delta_{q_{z}, 0} \delta_{q_{y}, 0} \sum_{i_{x}} \mathrm{e}^{\mathrm{i} q_{x} i_{x}}\left\langle\hat{S}_{i_{x}}^{\alpha}\right\rangle_{1 D} .
\end{aligned}
$$




\section{Appendix C: Symmetries}

\section{Heisenberg models}

For all the considered Heisenberg models, we have $\hat{H}=\left(\bigotimes_{i} \hat{U}_{i}\right) \hat{H}\left(\bigotimes_{i} \hat{U}_{i}\right)$, where all the $\hat{U}_{i}$ implement the same spin rotation. This implies $\left\langle\hat{S}_{\boldsymbol{i}}^{\alpha}\right\rangle=0$ and $\left\langle\hat{S}_{\boldsymbol{i}}^{\alpha} \hat{S}_{\boldsymbol{j}}^{\beta}\right\rangle=\delta_{\alpha, \beta}\left\langle\hat{S}_{\boldsymbol{i}}^{z} \hat{S}_{\boldsymbol{j}}^{z}\right\rangle$. Hence,

$$
\begin{aligned}
E(\boldsymbol{q}) & =1-\sum_{\alpha, \beta} \frac{\delta_{\alpha, \beta}-\bar{q}_{\alpha} \bar{q}_{\beta}}{N C_{s}} \sum_{\boldsymbol{i}, \boldsymbol{j}} \mathrm{e}^{\mathrm{i} \boldsymbol{q}(\boldsymbol{i}-\boldsymbol{j})}\left\langle\hat{S}_{\boldsymbol{i}}^{\alpha} \hat{S}_{\boldsymbol{j}}^{\beta}\right\rangle \\
& =1-\frac{2}{N C_{s}} \sum_{\boldsymbol{i}, \boldsymbol{j}} \mathrm{e}^{\mathrm{i} \boldsymbol{q}(\boldsymbol{i}-\boldsymbol{j})}\left\langle\hat{S}_{\boldsymbol{i}}^{z} \hat{S}_{\boldsymbol{j}}^{z}\right\rangle .
\end{aligned}
$$

For the quasi-one- and two-dimensional system we have

$$
E(\boldsymbol{q})=1-\frac{2}{L C_{s}} \sum_{i_{x}, j_{x}} \mathrm{e}^{\mathrm{i} q_{x}\left(i_{x}-j_{x}\right)}\left\langle\hat{S}_{i_{x}}^{z} \hat{S}_{j_{x}}^{z}\right\rangle_{1 D}
$$

and

$$
E(\boldsymbol{q})=1-\frac{2}{L^{2} C_{s}} \sum_{\tilde{\boldsymbol{i}}, \tilde{\boldsymbol{j}}} \mathrm{e}^{\mathrm{i} \tilde{\boldsymbol{q}} \cdot(\tilde{\boldsymbol{i}}-\tilde{\boldsymbol{j}})}\left\langle\hat{S}_{\tilde{\boldsymbol{i}}}^{\alpha} \hat{S}_{\tilde{\boldsymbol{j}}}^{\beta}\right\rangle_{2 D}
$$

respectively.

\section{XY model}

The Hamiltonian of the quasi-one-dimensional dimensional XY model,

$$
\hat{H}=\sum_{\langle i, j\rangle} \delta_{i, j}^{d}\left[(1+\gamma) \hat{S}_{i}^{x} \hat{S}_{j}^{x}+(1-\gamma) \hat{S}_{\boldsymbol{i}}^{y} \hat{S}_{\boldsymbol{j}}^{y}\right]-h \sum_{\boldsymbol{i}} \hat{S}_{\boldsymbol{i}}^{z}
$$

is invariant under simultaneous rotation of all the spins around their $z$ axis by $\pi$ (which takes $\hat{S}_{i}^{x}$ to $-\hat{S}_{i}^{x}, \hat{S}_{i}^{y}$ to $-\hat{S}_{i}^{y}$, and leaves $\hat{S}_{\boldsymbol{i}}^{z}$ invariant), which implies $\left\langle\hat{S}_{\boldsymbol{i}}^{x}\right\rangle=\left\langle\hat{S}_{\boldsymbol{i}}^{y}\right\rangle=\left\langle\hat{S}_{\boldsymbol{i}}^{z} \hat{S}_{\boldsymbol{j}}^{x}\right\rangle=\left\langle\hat{S}_{\boldsymbol{i}}^{z} \hat{S}_{\boldsymbol{j}}^{y}\right\rangle=0$. Hence, for $\boldsymbol{q}$ such that $\bar{q}_{x} \bar{q}_{y}=0$, we find

$$
\begin{aligned}
E(\boldsymbol{q}) & =1-\sum_{\alpha, \beta} \frac{\delta_{\alpha, \beta}-\bar{q}_{\alpha} \bar{q}_{\beta}}{N C_{s}}\left\langle\hat{S}_{\alpha, \beta}(\boldsymbol{q})\right\rangle \\
& =1-\frac{1}{N C_{s}} \sum_{\alpha}\left(1-\bar{q}_{\alpha}^{2}\right)\left\langle\hat{S}_{\alpha, \alpha}(\boldsymbol{q})\right\rangle,
\end{aligned}
$$

where, assuming $\boldsymbol{q} \neq \mathbf{0}$ and using translational invariance (such that we may write $s_{z}=\left\langle\hat{S}_{\boldsymbol{i}}^{z}\right\rangle$ ),

$$
\begin{aligned}
\left\langle\hat{S}_{\alpha, \alpha}(\boldsymbol{q})\right\rangle & =\sum_{\boldsymbol{i}, \boldsymbol{j}} \mathrm{e}^{\mathrm{i} \boldsymbol{q} \cdot(\boldsymbol{i}-\boldsymbol{j})}\left\langle\hat{S}_{\boldsymbol{i}}^{\alpha} \hat{S}_{\boldsymbol{j}}^{\alpha}\right\rangle=\sum_{\boldsymbol{i}, \boldsymbol{j}} \mathrm{e}^{\mathrm{i} \boldsymbol{q} \cdot(\boldsymbol{i}-\boldsymbol{j})}\left(\left\langle\hat{S}_{\boldsymbol{i}}^{\alpha} \hat{S}_{\boldsymbol{j}}^{\alpha}\right\rangle-\left\langle\hat{S}_{\boldsymbol{i}}^{\alpha}\right\rangle\left\langle\hat{S}_{\boldsymbol{j}}^{\alpha}\right\rangle\right)+\sum_{\boldsymbol{i}, \boldsymbol{j}} \mathrm{e}^{\mathrm{i} \boldsymbol{q} \cdot(\boldsymbol{i}-\boldsymbol{j})}\left\langle\hat{S}_{\boldsymbol{i}}^{\alpha}\right\rangle\left\langle\hat{S}_{\boldsymbol{j}}^{\alpha}\right\rangle \\
& =\sum_{\boldsymbol{i}, \boldsymbol{j}} \mathrm{e}^{\mathrm{i} \boldsymbol{q} \cdot(\boldsymbol{i}-\boldsymbol{j})} \delta_{\boldsymbol{i} \boldsymbol{j}}^{d}\left(\left\langle\hat{S}_{\boldsymbol{i}}^{\alpha} \hat{S}_{\boldsymbol{j}}^{\alpha}\right\rangle-\left\langle\hat{S}_{\boldsymbol{i}}^{\alpha}\right\rangle\left\langle\hat{S}_{\boldsymbol{j}}^{\alpha}\right\rangle\right)+\delta_{\alpha, z} s_{z}^{2} N^{2} \delta_{\boldsymbol{q}, \boldsymbol{0}} \\
& =\sum_{\boldsymbol{i}, \boldsymbol{j}} \mathrm{e}^{\mathrm{i} q_{d}\left(i_{d}-j_{d}\right)} \delta_{\boldsymbol{i}, \boldsymbol{j}}^{d}\left(\left\langle\hat{S}_{\boldsymbol{i}}^{\alpha} \hat{S}_{\boldsymbol{j}}^{\alpha}\right\rangle-\left\langle\hat{S}_{\boldsymbol{i}}^{\alpha}\right\rangle\left\langle\hat{S}_{\boldsymbol{j}}^{\alpha}\right\rangle\right) \\
& =L^{2} \sum_{i, j} \mathrm{e}^{\mathrm{i} q_{d}(i-j)}\left(\left\langle\hat{S}_{i}^{\alpha} \hat{S}_{j}^{\alpha}\right\rangle_{1 D}-\left\langle\hat{S}_{i}^{\alpha}\right\rangle_{1 D}\left\langle\hat{S}_{j}^{\alpha}\right\rangle_{1 D}\right)=: L^{2} \sum_{i, j} c_{i-j}^{\alpha}\left(q_{d}\right) .
\end{aligned}
$$

Due to translational invariance, we have $c_{l}^{\alpha}(q)=c_{l+L}^{\alpha}(q)=c_{l-L}^{\alpha}(q)=\left(c_{-l}^{\alpha}(q)\right)^{*}$, and hence for $L$ even,

$$
\sum_{i, j} c_{i-j}^{\alpha}(q)=L c_{0}^{\alpha}(q)+L c_{L / 2}^{\alpha}(q)+2 L \sum_{l=1}^{L / 2-1} \Re\left[c_{l}^{\alpha}(q)\right]=L\left(\frac{1}{4}-\delta_{\alpha, z} s_{z}^{2}\right)+L c_{L / 2}^{\alpha}(q)+2 L \sum_{l=1}^{L / 2-1} \Re\left[c_{l}^{\alpha}(q)\right]
$$


i.e.,

$$
E(\boldsymbol{q})=-1+4 s_{z}^{2}\left(1-\bar{q}_{z}^{2}\right)-4 \sum_{\alpha}\left(1-\bar{q}_{\alpha}^{2}\right)\left(c_{L / 2}^{\alpha}\left(q_{d}\right)+2 \sum_{l=1}^{L / 2-1} \Re\left[c_{l}^{\alpha}\left(q_{d}\right)\right]\right) .
$$

For the correlations functions, we use the results of [54] ( $1 \leq l \leq L / 2)$,

$$
\mathrm{e}^{-\mathrm{i} q l} c_{l}^{x}(q)=\frac{1}{4}\left|\begin{array}{cccc}
G_{-1} & G_{-2} & \cdots & G_{-l} \\
G_{0} & G_{-1} & \cdots & G_{-l+1} \\
\vdots & \vdots & \ddots & \vdots \\
G_{l-2} & G_{l-3} & \cdots & G_{-1}
\end{array}\right|, \quad \mathrm{e}^{-\mathrm{i} q l} c_{l}^{y}(q)=\frac{1}{4}\left|\begin{array}{cccc}
G_{1} & G_{0} & \cdots & G_{-l+2} \\
G_{2} & G_{1} & \cdots & G_{-l+3} \\
\vdots & \vdots & \ddots & \vdots \\
G_{l} & G_{l-1} & \cdots & G_{1}
\end{array}\right|, \quad \mathrm{e}^{-\mathrm{i} q l} c_{l}^{z}(q)=-\frac{1}{4} G_{l} G_{-l}
$$

where, for $L \rightarrow \infty$,

$$
\begin{aligned}
s_{z} & =\frac{1}{2 \pi} \int_{0}^{\pi} \mathrm{d} \phi \frac{\tanh [\beta \Lambda(\phi) / 2]}{\Lambda(\phi)}[h-\cos (\phi)], \\
G_{l} & =\frac{1}{\pi} \int_{0}^{\pi} \mathrm{d} \phi \frac{\tanh [\beta \Lambda(\phi) / 2]}{\Lambda(\phi)} \cos (\phi l)[h-\cos (\phi)]+\frac{\gamma}{\pi} \int_{0}^{\pi} \mathrm{d} \phi \frac{\tanh [\beta \Lambda(\phi) / 2]}{\Lambda(\phi)} \sin (\phi l) \sin (\phi), \\
\Lambda(\phi) & =\sqrt{\gamma^{2} \sin ^{2}(\phi)+(h-\cos (\phi))^{2}} .
\end{aligned}
$$

